\title{
Rhinolithiasis: A forgotten cause of nasal obstruction
}

\author{
Balouki Marouane $^{1 *}$, Zalagh Mohammed ${ }^{1}$, Benchafai Ilias ${ }^{2}$, Errami Noureddine ${ }^{1}$, Hemmaoui Bouchaib ${ }^{1}$ Jahidi Ali $^{1}$ and Benariba Fouad $^{1}$ \\ ${ }^{1}$ Department of Otorhinolaryngology, Mohammed V Military Teaching Hospital, Mohammed V-Souissi University, Hay Ryad 10100, Rabat, Morocco \\ ${ }^{2}$ Department of Otorhinolaryngology, 5th military hospital, Guelmim, Morocco
}

\begin{abstract}
\section{Introduction}

Rhinolithiasis is a rarely seen pathology, corresponding to the presence of a mineral mass in the nasal cavities, first described by Bertholin in 1654 [1]. Its pathogenesis is not clear, it remains disputed.

The development of these calcifications in the nose is asymptomatic at the beginning and leads to a chronic nasal obstruction after a long silent evolution. Nasal endoscopy is the examination of choice to visualize these foreign bodies [2]. Nasal CT is recommended before the surgery to assess and evaluate the size and location of the rhinolith and to rule out any associated pathologies with rhinolithiasis. We will report the case of a patient followed for chronic unilateral nasal obstruction, who was treated with endoscopic surgical removal of the stone in the operating room under general anesthesia.
\end{abstract}

Rhinolithiasis are foreign bodies that develop in the nasal cavities according to a poorly understood physicochemical mechanism. At first, they are asymptomatic, but once they are large, they give nasal obstruction with all the possible complications that can make it more difficult surgical management. we are going to present the case of a patient presenting with a rhinolithiasis discovered following a chronic nasal obstruction and whose endoscopy allowed the diagnosis and the extraction.

\section{Case report}

It is a 42-year-old male with no disease in the history of his medical records, presented with chronic unilateral right nasal obstruction without any symptoms of rhinorrhea, nor epistaxis nor a history of intranasal foreign body. The nasal obstruction was resistant to the usual medical treatment therefore a rhinoscopy and nasal endoscopy was preformed, which revealed a hard-intranasal mass below the middle turbinate in the right nasal fossa of yellowish color on a normal nasal mucosa (Figure 1).

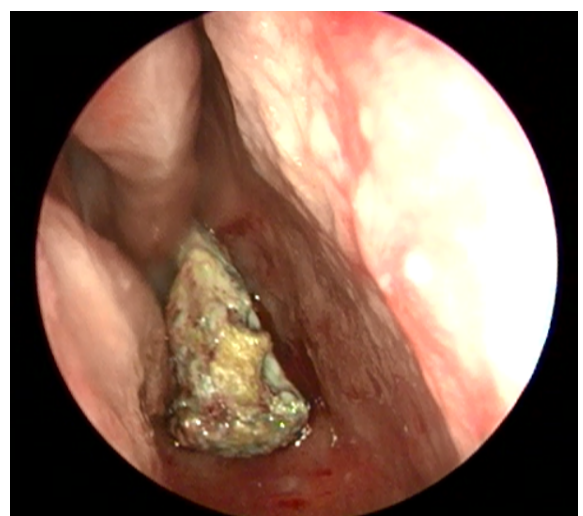

Figure 1. An endoscopic view (endoscope $0^{\circ}$ ) showing the macroscopic appearance of rhinolithiasis in the right nasal cavity

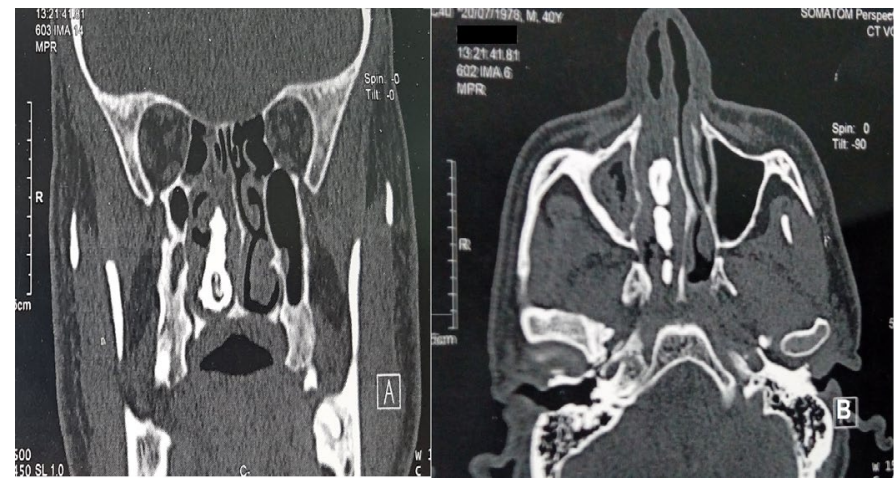

Figure 2. Naso-sinus CT, on coronal (A) and on axial views, bone windows: foreign body of calcium density, located in the nasal fossa, interposed between the inferior turbinate and the nasal septum

In order to better visualize the posterior extent of the mass and its contact with the mucosa, a maxillofacial CT scan was ordered, revealing a foreign body of calcium density in the right nasal cavity measuring 47/27/13 mm with an adjacent mucosal thickening. Associated with a right maxillary sinusitis and a right deviation of the nasal septum (Figure 2). Endoscopic removal of the rhinolith was performed under general anesthesia. histological evaluation of all the stone revealed acellular calcifications (calcium) consistent with rhinolithiasis.

\section{Discussion and conclusion}

The exact etiology and pathogenesis of intranasal rhinolithiasis is not completely understood [3]. It could be a chronic inflammation caused by a nasal foreign body acting as a nidus, with progressive precipitation of mineral salts (calcium, phosphorus, magnesium)

${ }^{\star}$ Correspondence to: Balouki Marouane, Department of Otorhinolaryngology, Mohammed V Military Teaching Hospital, Mohammed V-Souissi University, Hay Ryad 10100, Rabat, Morocco, Tel: +212696325410,E-mail: dr.baloukimaroune@ gmail.com

Key words: rhinolithiasis, nasal obstruction, foreign body, endoscopy

Received: September 14, 2020; Accepted: October 02, 2020; Published: October 05,2020 
leading to stone formation. This foreign body can be of endogenous origin (bone fragments, ectopic teeth, nasal epithelial debris) or exogenous (cotton wool, seeds, etc.). Other anatomical explanations have also been proposed: deviation of the nasal septum, bone spur raising the possibility of stagnation in the nose and thus extending the precipitation of nasal debris and foreign bodies. Another possibility is lack of mucociliary clearance resulting in stagnation of the exogenous debris that will act as a holding platform for the buildup of such stone [4].

The presenting symptoms of rhinolithiasis depend on the size of the stones, when they are still small, they are asymptomatic and diagnosed accidentally during a routine examination. Once the stone is big enough, it can give chronic nasal obstruction in the first place in addition to other non-specific symptoms that may appear namely epistaxis, swelling of the nose or face, anosmia, epiphora, and headache [5]. The goal of Nasal endoscopy is to visualize the nasal lesions and to make a possible biopsy or completely an extraction if it is possible [6], the maxillofacial scan is systematic to carry out a cartography of the lesions and to eliminate the differential diagnoses which are: calcified polyps, odontomas, granulomatous diseases, local osteomyelitis, osteomas, chondrosarcomas [7].

Usually, the extraction of rhinoliths is easy, through the anterior nostrils, in masse or in pieces under local or general anesthesia. If they are large, they can be moved back into the nasopharynx and removed transoral. In our case, removal through the anterior nostrils was easy, the rhinolith was split into pieces due to its big size which prevented mass removal.

In conclusion, rhinolithiasis could present with a wide spectrum of symptoms and signs and might be overlooked or mistaken for other diagnosis. It should be suspected in a patient with a long unilateral nasal obstruction, the treatment is easy and must be rapid to avoid certain complications like nasal deformity, nasal phlegmon, orbital abscess, meningitis. A histological evaluation of the removed lithiasis is required.

\section{Ethical approval}

The manuscript respects ethical publication standards, it does not contain any personal information of patients.

\section{Acknowledgment}

A special thank you to all the doctors who participated in the data collection via the survey form, and a sincere gratitude to the paramedical staff (nurses and caregivers) of the ENT department who participated in adequate patient care.

\section{References}

1. Kharoubi S (2008) General review of rhinolithiases. Ann Otolaryngol Chir CervicoFac 125: 11-17. [Crossref]

2. Edderai M, Saouab R, Boumdin H, Jidal M, El Fenni J, et al. (2019) La rhinolithiase savoir y penser devant une obstruction nasale chronique unilatérale. $J$ d'imagerie diagnostique Interv 2: 94-96.

3. Hadi U, Ghossaini S ZG (2002) Rhino- lithiasis: a forgotten entity. Otolaryngol Head Neck Surg 126: 48-51. [Crossref]

4. Zalagh M, Akhaddar A, Benariba F (2012) Chronic rhinorrhea revealing an actinomycotic rhinolithiasis with ectopic tooth. Int J Oral Maxillofac Surg 41: 297279. [Crossref]

5. Orhan K, Kocyigit D, Kisnisci R, Paksoy CS (2006) Rhinolithiasis: An uncommon entity of the nasal cavity. Oral Surg Oral Med Oral Pathol Oral Radiol Endod 101: 28-32.

6. Özkan Ö, Bingöl F, Budak A (2013) The importance of endoscopy in the diagnosis and treatment of rhinolithiasis: A case report. $J$ Acute Dis 2: 70-72.

7. Balatsouras DG, Eliopoulos P, Kaberos A, Economou C (2002) Rhinolithiasis: An unusual cause of nasal obstruction. Rhinology 40: 162-164. [Crossref]

Copyright: (C2020 Marouane B. This is an open-access article distributed under the terms of the Creative Commons Attribution License, which permits unrestricted use, distribution, and reproduction in any medium, provided the original author and source are credited. 